\title{
NGHIÊN CÚU QUY TRÌNH CÔNG NGHÊ THÀNH LẬP BẢN ĐỒ THÀNH PHỐ 3D TRÊN NỀN WEB
}

\author{
MAI ĐİNH SINH(1), VƯONG TRỌNG KHA ${ }^{(2)}$, TRỊNH LÊ HÙNG(1) \\ ${ }^{(1)}$ Học viện Kỹ thuật Quân sự, Hà Nội, Việt Nam \\ ${ }^{(2)}$ Truò̀ng Đại học Mỏ Địa chất, Hà Nội, Việt Nam
}

\section{Tóm tắt:}

Bài báo trình bày về phương pháp và quy trình thành lập bản đồ thành phố ảo $3 D$ trên nền Web. Các bước chính trong quy trình bao gồm thu thập thông tin không gian địa lý, xây dựng mô hình $3 D$ và trục quan hóa dư liệu $3 D$ thành phố trên nền web. Thử nghiệm xây dưng úng dụng thành phố ảo $3 D$ trên nền Web dựa trên nền tảng $3 D$ city $D B$, là gói phần mềm mã nguồn mở cho phép luu trũ̃, quản lý và hiển thị hiệu quả các mô hình thành phố 3D ở định dạng CityGML một cách hiệu quả. Kết quả thủ nghiệm cho thấy việc ứng dụng mã nguồn mở để thành lập các bản đồ ảo $3 D$ trên nền Web có nhiều ưu điểm nhu tiết kiệm thời gian, chi phí và tận dụng được nhiều nguồn dũ liệu hiện có trong việc xây dụng các ứng dụng khai thác dũ liệu thành phố ảo 3D.

\section{Mở đầu}

Mô hình dữ liệu 3D được coi là một công cụ để thể hiện các đối tượng 3D trong thực tế, cho phép mô hình hóa hiệu quả bằng cách hiển thị chiều địa lý thứ ba [4]. Với sự phát triển mạnh mẽ của công nghệ mạng, các ứng dụng bản đồ dựa trên nền tảng web đã chứng tỏ được ưu thế trong rất nhiều các bài toán. Việc sử dụng hiệu quả mô hình 3D GIS trong quá trình ra quyết định là một cách truyền đạt ý tưởng rất nhanh chóng, giúp đưa ra quyết định tốt hơn [5]. Tuy nhiên, hầu hết các ứng dụng 3D GIS tại Việt Nam hiện nay đều gặp khó khăn trong việc biểu diễn dữ liệu mô hình 3D trên nền web. Vì vậy, việc đề xuất quy trình xây dựng bản đồ $3 \mathrm{D}$ nói chung và bản đồ thành phố ảo $3 \mathrm{D}$ trên nền web là bài toán có tính khoa học và thực tiễn cao [6].

Các công đoạn chính để xây dựng một hệ thống thông tin bản đồ 3D bao gồm: thu thập dữ liệu $3 \mathrm{D}$, mô hình hóa và trực quan hóa dữ liệu 3D, quản lý dữ liệu 3D [7]. Việc thiết lập môi trường $3 \mathrm{DC}$ ityDB yêu cầu cài đặt chạy một số công cụ phần mềm có sẵn hoàn toàn miễn phí. Gói phần mềm 3DCityDB đi kèm với một bộ tập lệnh SQL để tạo lược đồ quan hệ trên hệ thống cơ sở dữ liệu không gian (PostgreSQL/PostGIS) và với một nhóm tập lệnh $\mathrm{PL} / \mathrm{pgSQL}$ để quản lý mô hình thành phố $3 \mathrm{D}$ được lưu trữ trong cơ sở dữ liệu [8]. Bản đồ 3D là một trong những chủ đề rất được quan tâm trong các hệ thống GIS (hệ thống thông tin địa lý) hiện nay. Có nhiều nỗ lực nghiên cứu trong lĩnh vực phát triển bản đồ 3D. Brooks và Whalley [1] đã trình bày một hệ thống GIS cho phép hiển thị $2 \mathrm{D}$ và $3 \mathrm{D}$ từ cùng một dữ liệu không gian. Nhiều lớp thông tin được chuyển đổi liên tục giữa các chế độ $2 \mathrm{D}$ và $3 \mathrm{D}$ dưới sự điều khiển của người dùng và tương tác trực tiếp trên mô hình địa hình số. GIS 3D có thể được sử dụng để phân tích độ dốc và điều tra các đường dẫn nước và vị trí của các tảng đá có thể rơi xuống. Shehata và Koshak [2] đã xây dựng một mô hình 3D để phân tích vị trí, độ dốc, loại và quy mô của những rủi ro thiên nhiên có thể xảy ra, từ đó có thể đưa ra các dự báo sớm nhằm hạn chế những thiệt hại do thiên tai gây ra.

Vì vậy, việc nghiên cứu các ứng dụng thành lập bản đồ 3D trên nền Web là xu hướng tất yếu và có nhiều ý nghĩa thực tiễn. Các công nghệ $3 \mathrm{D}$ GIS có ưu điểm trong việc cung cấp các chức năng cần thiết cho việc quản lý dữ liệu, quy trình 
công việc của người dùng và các quá trình liên quan đến hạ tầng đô thị. Nội dung nghiên cứu tiếp theo sẽ bao gồm phương pháp và quy trình công nghệ, thực nghiệm, thảo luận.

\section{Cơ sở khoa học}

Hiện nay nhu cầu sử dụng và khai thác thông tin trên các ứng dụng Web ngày càng trở nên phổ biến. Các hệ thống GIS $2 \mathrm{D}$ và $3 \mathrm{D}$ trên các nền tảng desktop đã bộc lộ những hạn chế trong việc chia sẻ và khai thác thông tin, dữ liệu gồm nhiều định dạng khác nhau và việc quản lý đang phân tán, dẫn đến nhu cầu cấp thiết trong việc nghiên cứu và đề xuất các giải pháp trực tuyến có thể chia sẻ và khai thác thông tin địa không gian một cách hiệu quả, nhanh chóng. Hơn nữa, do quá trình đô thị hóa diễn ra nhanh chóng, dẫn đến nhu cầu lớn trong việc khai thác các thông tin địa lý để xây dựng các thành phố thông minh phục vụ du lịch, kinh tế, xã hội, an ninh quốc phòng và quy hoạch đô thị, ứng phó với các sự cố thiên tai, môi trường,...

Tuy vậy, các kết quả nghiên cứu thành lập bản đồ $3 \mathrm{D}$ Web vẫn rất hạn chế, chủ yếu sử dụng phần mềm để mô phỏng nhưng việc tương tác và khai thác các thông tin trên vẫn còn rất hạn chế. Các nghiên cứu đã công bố ở Việt nam vẫn chưa đủ cơ sở pháp lý để ban hành các tiêu chuẩn và quy chuẩn cho việc xây dựng và thành lập bản đồ 3D Web. Về mặt công nghệ, với sự phát triển mạnh mẽ của công nghệ thông tin, nhiều chuẩn mở thông tin địa lý ra đời nhằm đáp ứng nhu cầu xây dựng các bản đồ $3 \mathrm{D}$ trên nền Web cho phép trao đổi dữ liệu không gian 3D trong môi trường web. Về dữ liệu thông tin địa lý, tại Việt Nam dữ liệu nền địa lý $2 \mathrm{D}$ đã phủ kín cả nước với tiêu chuẩn và quy chuẩn quốc gia, đây là một trong những nguồn dữ liệu quan trọng làm đầu vào cho các mô hình và bản đồ $3 \mathrm{D}$.

Những vấn đề trên là cơ sở khoa học vững chắc, để các tác giả nghiên cứu đề xuất quy trình thành lập bản đồ thành phố 3D trên nền Web.

\subsection{Chuẩn dũ̃ liệu}

Có nhiều chuẩn dữ liệu được sử dụng để biểu diễn dữ liệu 3D, trong số đó, chuẩn CityGML là chuẩn dưới dạng ngôn ngữ eXtensible MarkUp Language (XML) được xây dựng thành tiêu chuẩn quốc tế do Open GIS Consortium (OGC) đề xuất với mục đích thành lập và trao đổi dữ liệu không gian đô thị 3 chiều. Trong CityGML, các đối tượng địa lý $3 \mathrm{D}$ trong đô thị được định nghĩa về mặt hình học, cấu trúc hình học (topology), các tính chất chuyên đề cũng như hình dáng bên ngoài [3]. Các định nghĩa này cho phép mã hóa các đối tượng địa lý 3D trong đô thị phục vụ các mục đích như quy hoạch đô thị, định vị, mô phỏng các tình huống môi trường và quản lý hạ tầng đô thị.

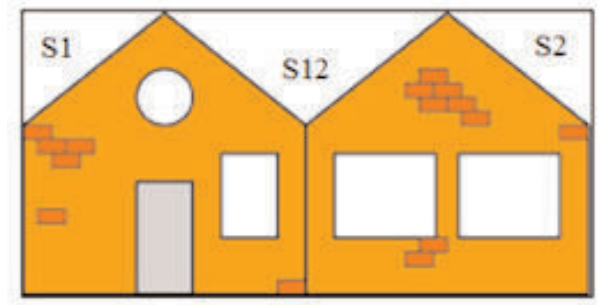

\section{Hình 1: Mối quan hệ giũa hai đối tượng khi có} điểm chung

Các đối tượng hình học trong CityGML có quan hệ hình học (topology) tương đối đơn giản, có thể sở hữu chung một đối tượng hình học là thành phần của nó. Ví dụ như hai hình khối đặc đại diện cho nhà $(\mathrm{S} 1)$ và nhà $(\mathrm{S} 2)$ có thể chung nhau một mặt phẳng đại diện cho bức tường chung (S12) giữa hai đối tượng này (Hình 1). Việc lưu trữ này có thể tiết kiệm được không gian lưu trữ, giảm thiểu được thời gian hiển thị mô hình. Trong CityGML, các đối tượng địa lý 3D được định nghĩa về mặt hình học, topology, các tính chất chuyên đề cũng như hình dáng bên ngoài. Các định nghĩa này cho phép mã hóa các đối tượng địa lý $3 \mathrm{D}$ trong thành phố phục vụ các mục đích như quy hoạch đô thị, định vị, mô phỏng các tình huống môi trường và quản lý hạ tầng đô thị. Sau đó, các phương pháp khái quát hóa được nghiên cứu và điều chỉnh để tạo ra các cảnh thành phố $3 \mathrm{D}$ ở các quy mô khác nhau một cách linh hoạt. Nhiều cấu trúc đại diện được thiết kế để bảo tồn các kết quả tổng quát hóa ở mức 
độ khác nhau.

Đối tượng trong CityGML được biểu diễn theo nguyên tắc đa tỷ lệ với các cấp độ chi tiết khác nhau. Các đối tượng không gian được chia thành 5 mức độ chi tiết (Level of Detail) khác nhau bao gồm LoD0, LoD1, LoD2, LoD3 và LoD4. Hình 2 là ví dụ về một đối tượng không gian là một ngôi nhà được hiển thị ở các cấp độ chi tiết khác nhau. Trong các cấp độ chi tiết, cấp độ chi tiết LoD0 là cấp độ tương đương với dữ liệu 2D (bao gồm các đường viền chân nhà). Cấp độ chi tiết LoD1 sẽ hiển thị mỗi khối nhà bằng một hình khối đặc đơn giản bằng cách dâng cao đường viên chân nhà lên một độ cao nhất định. Cấp độ chi tiết LoD2 sẽ bổ sung thêm phần mái nhà so với cấp độ chi tiết LoD1. Ở cấp độ chi tiết LoD3, các phần của ngôi nhà sẽ được bổ sung như ống khói, các cửa sổ, cửa ra vào, v.v... Ở cấp độ chi tiết cao nhất LoD4, mỗi ngôi nhà có thể hiển thị cả không gian bên trong nhà, các đồ vật, nội thất bên trong của ngôi nhà.
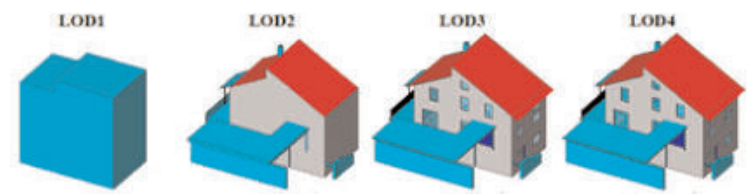

Hình 2: Các cấp độ chi tiết của đối tượng $3 D$

Các đối tượng không gian 3D có thể được tạo thành từ bề mặt với các chất liệu khác nhau, chẳng hạn một ngôi nhà có thể có mái ngói, mái tôn, mái xi măng,... Mô hình 3D của đối tượng thể hiện được các đặc tính này bằng cách xây dựng các bề mặt hiển thị trong phần định nghĩa đối tượng CityGML. Bề mặt hiển thị này có thể có thể xây dựng bằng chụp ảnh thực, hoặc tạo ra bằng các hình dạng hoa văn. CityGML có cơ chế cho phép xây dựng các đối tượng mở rộng ngoài các đối tượng không gian. Ngoài việc định nghĩa thêm các đối tượng thì người sử dụng cũng có thể định nghĩa thêm các thuộc tính của mỗi đối tượng có sẵn. Ví dụ để phục vụ mục đích quản lý đô thị thì có thể thêm các thuộc tính cho đối tượng nhà như năm xây dựng, loại nhà, v.v...

\subsection{Quy trình công nghệ}

Hình 3 mô tả quy trình thành lập bản đồ thành phố 3D trên nền Web. Các bước chính của quy trình bao gồm: Lập kế hoạch và thu thập số liệu; Chuẩn hóa và xây dựng dữ liệu; Tích hợp các lớp dữ liệu; Trực quan hóa dữ liệu trên nền Web.

a. Lập kế hoạch và thu thập dũ liệ: Từ yêu cầu của bài toán, tiến hành thu thập dữ liệu về địa hình, dữ liệu ảnh nền và dữ liệu về các lớp đối tượng trên nền địa hình, đo đạc và thu thập hình ảnh các đối tượng ngoài thực địa. Dũ liệu địa hình và ảnh vệ tinh được lấy từ các nguồn dữ liệu có sẵn trên internet.

b. Chuẩn hóa và xây dựng dũ liệu: Dữ liệu sau khi thu thập thường ở dạng thô, vì vậy cần phải chuẩn hóa, số hóa, hoặc chuyển đổi về các định dạng phù hợp với các chuẩn để tích hợp và hiển thị lên nền Web. Trước khi sử dụng cần một bước kiểm tra chất lượng dữ liệu.

c. Tích hợp các lớp dũ liệu: Dữ liệu sau khi hiển thị trên nền Web cần được tích hợp dữ liệu mô hình và thông tin về các đối tượng, tích hợp các chức năng tương tác và khai thác các thông tin của các đối tượng trên bản đồ. (Xem hình 3,4)

d. Trục quan hóa dĩ liệu trên nền Web: Đây là một bước quan trọng để hiển thị, trình bày và trực quan hóa thông tin một cách hiệu quả dữ liệu bản đồ trên nền Web đến với người dùng. Bao gồm mô hình số địa hình, mô hình $3 \mathrm{D}$ các lớp đối tượng trên bản đồ, các bảng biểu, đồ thị,... Mục đích là từ dữ liệu thô biến thành những thông tin được thể hiện một cách trực quan, dễ quan sát, dễ hiểu, để truyền đạt những hiểu biết đầy đủ về dữ liệu đến người sử dụng.

\subsection{Kiến trúc tổng quát của hệ thống $3 \mathrm{D} \mathrm{Web}$}

Hình 4 mô tả kiến trúc tổng quát của hệ thống 3D GIS trên nền Web, kiến trúc này bao gồm 2 thành phần chính là máy chủ $3 \mathrm{D}$ Web và máy khách 3D Web.

Máy khách 3D Web: Là nơi hiển thị các dữ liệu từ máy chủ $3 \mathrm{D}$ Web, hiển thị trên nền web và các công cụ cho phép người sử dụng có thể tương tác, thao tác trên dữ liệu như: Hiển thị thuộc tính, điều khiển lớp bản đồ, điều khiển đối 


\section{Nghiên cứu - Úng dụng}

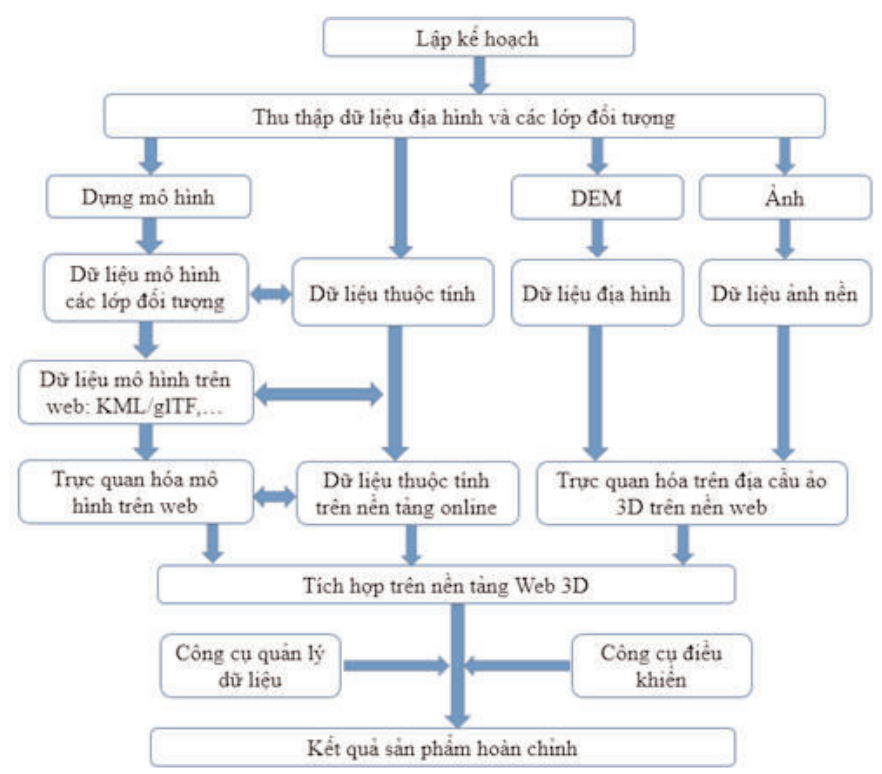

Hình 3: Quy trình công nghệ thành lập bản đồ thành phố ảo $3 D$ trên nền web



Hình 4: Kiến trúc tổng quát của hệ thống 3D GIS trên nền Web

tượng, các chức năng tương tác với bản đồ, các chức năng thêm, sửa, xóa,...

Máy chủ $3 D$ Web: Là nơi lưu trữ dữ liệu và khi có ưu cầu từ máy khác, máy chủ $3 \mathrm{D}$ web sẽ thực hiện truy vấn dữ liệu mô hình và dữ liệu thuộc tính, trực quan hóa mô hình trước khi hiển thị trên nền web ở máy khách. Máy chủ 3D Web cũng quản lý và lưu trữ dữ liệu mô hình số độ cao và dữ liệu ảnh nền.

\section{Thực nghiệm}

\subsection{Khu vục thử nghiệm và công nghệ sử dụng}

Khu vục thưc nghiệm: Trong thử nghiệm này, bài báo sử dụng dữ liệu mở được cung cấp miê̂n phí từ dự án 3DcityDB (http://3dcitydb.org) để làm dữ liệu đã thu thập dạng CityGML. Khu vực xây dựng đô thị $3 \mathrm{D}$ là một vùng thuộc trung tâm thành phố Berlin (Hình 6).

Công nghệ sử dụng: Hệ điều hành Microsoft 
Windows 10, máy tính Dell core i5, 1.7GHz, $16 \mathrm{~Gb}$ RAM và $2 \mathrm{~Gb}$ Card Geofore NVIDIA. Hệ quản trị cơ sở dữ liệu PostgreSQL 10, cùng các extension giúp lưu trữ và xử lý các dữ liệu dạng vị trí, hình học và kết cấu địa lý: $\operatorname{pgAdmin} 4$, PostGIS, pgRouting, GEOS, SFCGAL. Sử dụng công cụ 3DCityDB-Importer-Exporter để import dữ liệu từ dạng CityGML vào cơ sở dữ liệu. Đây là công cụ mã nguồn mở của dự án 3D City $\mathrm{DB}$, nó giúp dễ dàng nhập và xuất dữ liệu vào cơ sở dữ liệu dùng PostgreSQL. Đồng thời cũng là công cụ để trích xuất dữ liệu từ cơ sở dữ liệu ra một số dạng dữ liệu như KML/COLLADA/gITF. Sử dụng dữ liệu ảnh nền được cung cấp miễn phí trên internet. Sử dụng gói phần mềm 3DCityDB làm hệ thống hiển thị mô hình đô thị $3 \mathrm{D}$. Đây là một sản phẩm mã nguồn mở của tổ chức 3D City $\mathrm{DB}$, có thể download tại: https://github.com/3dcitydb/3dcitydb-web-map. Một số thư viện hỗ trợ máy chủ web và máy khách web trong việc hiển thị và truy vấn dữ liệu bao gồm Java 8, Node.js.

\subsection{Kết quả và thảo luận}

Chương trình được cài đặt trong thư mục 3DcityMap được đặt tại ổ $\mathbf{C}: 1$. Để chạy chương trình, trước hết cần kích hoạt server: C:|3DCityMap>node server.js với cổng mặc định là 8000 . Mở đường dẫn https://localh o s t : $8000 / 3 \mathrm{~d} \mathrm{c} \mathrm{i} \mathrm{t} \mathrm{y} \mathrm{d} \mathrm{b} \mathrm{-} \mathrm{w} \mathrm{e} \mathrm{b} \mathrm{-}$ map/3dwebclient/index.html, khi đó giao diện chương trình Bản đồ Thành phố 3D trên nền Web sẽ hiển thị như hình 5 .

Hình 5 là giao diện web thành phố $3 \mathrm{D}$. Với các chức năng cơ bản như phóng to, thu nhỏ, di chuyển, xoay. Các chức năng hiển thị nền địa hình, chức năng quản lý các lớp dữ liệu mô hình. Hình 6 là khu vực thử nghệm với dữ liệu nền là dữ liệu OpenStreetMap. (Xem hình 5,6)

Hình 7 mô tả chức năng quản lý và hiển thị nguồn dữ liệu nền bản đồ, có nhiều nguồn dữ liệu có thể được khai thác miễn phí như ESRI World Imagery, ESRI World Street Map, OpenStreetMap, Natural Earth II,... và các chức năng thao tác cơ bản với bản đồ như phóng to, thu nhỏ, di chuyển, xoay,... Phần chức năng chính cho phép người dùng có thể thêm và cấu hình các lớp dữ liệu. Tạo các hiệu ứng cho các đối tượng trên bề mặt địa hình như tô bóng, highlight, chụp ảnh màn hình, in ấn,... Hình 8 là hình ảnh thành phố $3 \mathrm{D}$ được hiển thị ở mức LOD2. (Xem hinh 7,8,9)

Hình 9 là thanh công cụ cho phép thao tác nhanh với một số chức năng như tìm kiếm một địa điểm trên bản đồ, thay đổi dữ liệu ảnh nền, hiển thị tọa độ hiện tại... Từ kết quả thử nghiệm trên, có thể thấy rằng với quy trình được đề xuất ở trên, bài báo đã ứng dụng và xây dựng thành công bản đồ thành phố $3 \mathrm{D}$ trên nền Web. Với rất nhiều các phần mềm có thể xây dựng mô hình 3D hiện nay, hoàn toàn có thể xây dựng được dữ liệu đầy đủ của các thành phố. Từ đó dữ liệu này có thể quản lý tập trung trên các máy chủ 3D Web và quản lý, khai thác sử dụng trên nền máy khách 3D Web. Một số ưu, nhược điểm có thể kể đến như sau:

Uu điểm: Việc sử dụng nền tảng 3D Web mã nguồn mở, có thể giúp giảm thiểu thời gian phát triển các ứng dụng bản đồ 3D trên nền Web. Tận dụng được những công nghệ mới nhất với sự chung tay từ cộng đồng. Dễ dàng truy cập, hiển thị và trực quan hóa mô hình đô thị $3 \mathrm{D}$ nhanh chóng. Dễ dàng triển khai cho nhiều máy, chi phí triển khai thấp. Dữ liệu được tập trung nên dễ quản lý, cập nhật. Có thể thấy, giải pháp trực quan hoá đô thị $3 \mathrm{D}$ theo chuẩn CityGML có nhiều ưu điểm so với một số ứng dụng 3D GIS đã có.

Nhược điểm: Yêu cầu bảo mật với những thông tin nhạy cảm như thông tin về an ninhquốc phòng, thông tin về tài nguyên mật quốc gia,... Vì vậy, để có thể triển khai trong thực tế cần có sự đầu tư nghiên cứu để phát triển ứng dụng dựa trên mã nguồn mở với các yêu cầu bảo mật thông tin có thể đáp ứng được yêu cầu đặt ra. Đối với các bài toán trong quân sự, có thể tận dụng mạng thông tin quân sự để xây dựng ứng dụng 3D GIS trên nền Web phù hợp với yêu cầu và nhiệm vụ được giao. 


\section{Nghiên cúu - Úng dụng}



Hình 5: Giao diện chuơng trình và khu vục thư nghiệm



Hình 6: Khu vục thư nghiệm
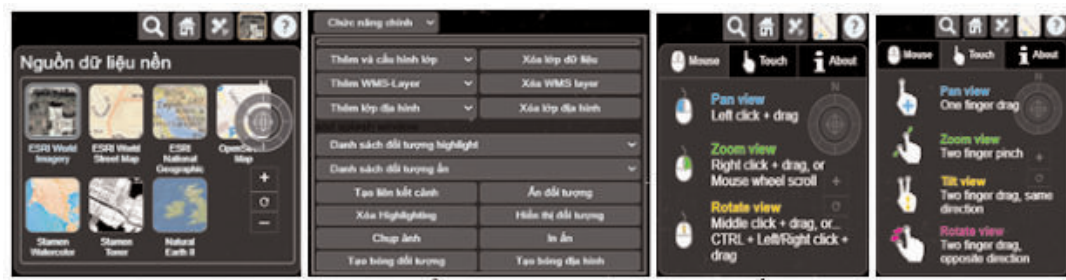

Hình 7: Dũ liệu nền và chức năng quản lý lớp đối tượng

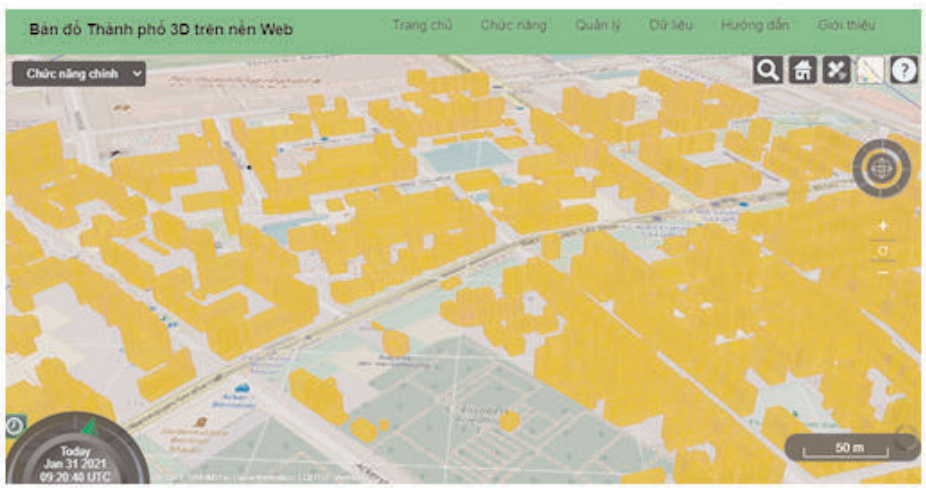

Hình 8: Dũ liệu mô hình khi hiển thị ở mức LOD2

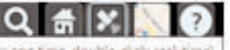

Hìn 9: Thanh công cu của chuoong trình 


\section{Kết luận}

Ứng dụng 3D GIS được phát triển nhằm đánh giá không gian đô thị một cách hiệu quả và cung cấp thông tin về quy hoạch đô thị cho cộng đồng địa phương, cung cấp cho các nhà thiết kế và quy hoạch đô thị một công cụ hữu ích để lập mô hình và phân tích. Ứng dụng này cho phép người dùng hình dung các thông tin quy hoạch đô thị phức tạp theo cách $3 \mathrm{D}$, để đánh giá công suất cho phép của khu nhà và mô phỏng các quy hoạch xây dựng của các thành phố. Với khả năng trực quan hóa và phân tích, 3D GIS được coi là một công cụ mạnh mẽ để giải quyết các vấn đề khác nhau mà các thành phố hiện đại phải đối mặt. $O$

\section{Tài liệu tham khảo}

[1]. S. Books, J.L. Whally, A 2D/3D hybrid geographical information system, Proceedings of ACM, GRAPHITE, Dunedin, Canada, 2005.

[2]. A.M. Shehata, N.A. Koshak, Using 3D GIS to Assess Environmental Hazards in Built Environmental, Journal of Al Alzhar University, Engineering Sector, Cairo, Egypt, 2006.

[3]. Christoph Blut, Timothy Blut \& Jörg Blankenbach (2019) CityGML goes mobile: application of large 3D CityGML models on smartphones, International Journal of Digital Earth, 12:1, 25-42.
[4]. Schilling, A., J. Bolling, and C. Nagel. 2016. "Using gITF for Streaming CityGML 3D City Models." (October 2015), 109-116.

[5]. Biljecki, F., J. Stoter, H. Ledoux, S. Zlatanova, and A. Çöltekin. 2015. "Applications of 3D City Models: State of the Art Review." ISPRS International Journal of Geo-Information 4 (4): 2842-2889.

[6]. Zhihang Yao, Claus Nagel, Felix Kunde, György Hudra, Philipp Willkomm, Andreas Donaubauer, Thomas Adolphi and Thomas H. Kolbe. 3DCityDB - a 3D geodatabase solution for the management, analysis, and visualization of semantic 3D city models based on CityGML. Open Geospatial Data, Software and Standards (2018) 3:5.

[7]. Blut C, Blut T, Blankenbach J. CityGML goes mobile: application of large 3D CityGML models on smartphones. International Journal of Digital Earth. 2017:1-18.

[8]. Beil C, Kolbe TH (2017) CityGML and the streets of New York - a proposal for detailed street space modelling. Proceedings of the 12th $3 D$ GeoInfo conference 2017, Melbourne, Australia, 26-27 October 2017. ISPRS Annals of the Photogrammetry, Remote Sensing and Spatial Information Sciences, Vol. IV-4/W5, pp 9-16. O

\section{Summary}

\section{Study of the technological process for building 3D city map on Web}

Mai Dinh Sinh, Trinh Le Hung

Military Technical Academy, Hanoi, Vietnam

\section{Vuong Trong Kha, Hanoi University of Mining and Geology, Hanoi, Vietnam}

With the strong development of science and technology, web-based mapping applications have shown superior advantages over traditional map representation methods. The paper discusses methods and steps taken to build a 3D geospatial information system. The main objective of this study is to propose the process of creating a 3D virtual city map on the Web. Key steps in the process include gathering geospatial information, building 3D models, and visualizing 3D city-based city data. Building 3D virtual city application on Web based on 3DCityDB platform. It is an open source software package that allows efficient storage, management, and display of 3D city models in CityGML format. The test results show that the application of open source to create 3D virtual maps on the Web has many advantages such as saving time, cost and utilizing many existing data sources in building applications. 3D virtual city data mining application. $\bigcirc$ 